\title{
Enfrentamento da violência doméstica e maus-tratos aos animais em tempo de COVID-19
}

\author{
Larissa Rachel Wolf \\ larissarwolf@hotmail.com \\ Fernanda Muraro de Alvarenga \\ feralvarenga1508@gmail.com \\ Letícia Hauptman \\ leticia.hauptman@gmail.com \\ Bruno Pedon Nunes \\ brunonunes@ufpr.br \\ Rita de Cassia Maria Garcia \\ ritamaria@ufpr.br
}

\section{Resumo}

0 Projeto de Extensão "Enfrentamento da Violência Doméstica e Maus-Tratos aos Animais em Tempo de COVID", possui como objetivo a promoção de ações para o entendimento da Teoria do Elo pela comunidade, a fim de criar uma rede para o enfrentamento à violência doméstica e maus-tratos aos animais no período de pandemia, através da promoção de medidas socioeducativas por meio das Redes Sociais, criação Website, curso e palestras Online e materiais didáticos. Todo conteúdo foi desenvolvido por 31 alunos extensionistas de diferentes áreas do Brasil. Com o desenvolvimento do projeto foi notável a importância da disseminação de tais informações para a comunidade de forma didática e holística.

\section{Palavras-chave}

Violência doméstica; COVID-19; Teoria do Elo. 


\section{Introdução}

Com o avanço rápido da pandemia COVID-19 pelo mundo, tornou-se necessário, como medida de prevenção da disseminação do vírus Sars-Cov-2 que as pessoas fiquem reclusas em seus lares, em isolamento social, o que provocou diversas mudanças de hábitos e as consequências atingiram não só a população, como também os animais domésticos. Uma das complicações do confinamento foi o aumento da violência doméstica, que afeta principalmente mulheres, crianças, idosos e os animais, uma vez que estes estão passando cada vez mais tempo ao lado do seu agressor.

O confinamento causado pela pandemia aumentou significativamente as agressões sofridas. Segundo os dados da Ouvidoria Nacional de Direitos Humanos (ONDH), houve um aumento de $37,58 \%$ de denúncias de violência contra a mulher registradas pelo Ligue 180 no mês de abril deste ano, comparado com 2019 (FEDERAL, 2020). 0 risco crescente de homicídios relacionados com a violência doméstica é também uma preocupação crescente, relatórios de homicídios por parceiros íntimos com ligações ao estresse ou outros fatores relacionados com a pandemia de Covid-19 continuam a surgir em todo o mundo (CAMPBELL, 2020).

A comunidade científica já reconhece a relação existente entre os maus-tratos aos animais e a violência interpessoal, chamada de "Teoria do Elo", visto que diversos estudos correlacionam esses casos e demonstram que o ciclo de violência envolvido parte de um adulto oriundo de um lar caótico e que foi dessensibilizado para as consequências da violência, já que este pode ter sido vítima desse ciclo ou possuir uma desordem de personalidade (ASCIONE et al., 1997; ASCIONE et al., 2010; BALKIN et al., 2013).

0 abuso de animais é um importante indicador de violência doméstica e comportamento criminoso (LOOKWOOD, 2000; MONSALVE et al., 2017). Desta forma, a detecção de maus-tratos aos animais é de grande importância, não só para a proteção de seres sencientes, mas também para a realização de intervenções que visam melhorar a qualidade de vida das famílias vulneráveis (MONSALVE et al., 2017) e diminuir os níveis de violência na sociedade (BENETATO; REISMAN; MCCOBB, 2011) 0 sistema jurídico geralmente ignora a relação humano-animal (ARKOW, 2020), e os casos de maus-tratos e outros tipos de delitos contra os animais são frequentemente banalizados e tratados como crimes menores e independentes dos delitos contra as pessoas (RANDALL, 2008; ARKOW, 2020).

No Brasil, existe legislação específica para proteger os animais dos maus-tratos (BRASIL, 1998). A Lei Federal 9.605, de 12 de fevereiro de 1998, dispõe sobre as sanções penais e administrativas derivadas de condutas e atividades lesivas ao meio ambiente e dá outras providências e que prevê, no capítulo V, destinado aos "Crimes Contra o Meio Ambiente", em sua 
Seção I, que define os "Crimes Contra a Fauna", o artigo 32, que tipifica: "Praticar ato de abuso, maus tratos, ferir ou mutilar animais silvestres, domésticos ou domesticados, nativos ou exóticos: Pena - detenção, de três meses a um ano, e multa".

Neste contexto de violência doméstica, no qual o animal também é inserido, é importante incluir profissionais capacitados para sua detecção e manejo preventivo e interventivo nos lares. Portanto, o médico veterinário dentro da Medicina Veterinária do Coletivo, área com uma abordagem multidisciplinar de questões que envolvem animais, seres humanos e meio ambiente, atua também de forma intersetorial e em parceria com instituições públicas, como universidades, ministério público e polícia civil, sendo um profissional importante para a promoção de segurança pública dentro das comunidades (MONSALVE et al., 2019).

Pesquisas e trabalhos na área são fundamentais para melhorar o diagnóstico e conhecer o cenário real dessa relação no país, buscando encontrar estratégias para o seu enfrentamento. Ações intersetoriais e multiprofissionais envolvendo instituições policiais, promotorias públicas, profissionais da assistência social, saúde, educação e meio ambiente (defesa e proteção animal) são de extrema importância para a quebra desse ciclo de violência.

Visto a importância dessa relação, o Projeto de Extensão "Enfrentamento da Violência Doméstica e Maus-Tratos aos Animais em Tempo de COVID" promove conteúdos interdisciplinares que visam a socioeducação da comunidade e dos participantes sobre tais assuntos através da Internet, para isso, conta com a participação de 28 voluntários, de diversas universidades do Brasil e diferentes cursos, três alunas bolsistas de Medicina Veterinária da UFPR e residentes de Medicina Veterinária do Coletivo da UFPR.

O objetivo geral do projeto é promover ações e propagar informações para o entendimento da Teoria do Elo pelos diferentes setores e profissionais, comunidade e alunos participantes, a fim de criar uma rede interdisciplinar e intersetorial para o enfrentamento da violência doméstica e maus-tratos aos animais no período de pandemia.

\section{Metodologia}

Foram realizadas medidas socioeducativas e interdisciplinares, com públicos-alvo e abordagens distintas, através de diferentes plataformas online, usando canais como Instagram, Facebook, blog da revista Clínica Veterinária, publicação de cartilhas e e-books na Biblioteca Virtual da Universidade Federal do Paraná (UFPR). Além disso, o projeto também promoveu o desenvolvimento de atividades de ensino de diversas modalidades. 
Com o auxílio de 5 alunas voluntárias foram produzidas 19 postagens, até então, para a plataforma Instagram, com o objetivo de informar a comunidade em geral. As publicações postadas no Instagram Medicina Veterinária do Coletivo (@mvcufpr) forneceram o entendimento, de forma simples, concisa e acessível, sobre temas relacionados à Violência Doméstica, Maustratos aos Animais, Teoria do Elo, Autoconhecimento e Zoonoses Urbanas.

Os assuntos abordados nas postagens foram: "Mas afinal, onde atua o Médico Veterinário do Coletivo?" (22/06); "Qual o papel do Médico Veterinário no bem-estar das pessoas e dos animais" (25/06); "Coronavírus em humanos e animais: Entenda as diferenças" (06/07); "Como a pandemia afeta os animais?" (11/07); “Teoria do Elo" (15/07); “Aumento da violência doméstica e familiar" (16/07); "Violência contra os animais" (21/07); "Principais Zoonoses urbanas: Leptospirose" (22/07); "Apresentação do curso Enfrentamento da violência doméstica e dos maus-tratos aos animais em tempos de COVID" (24/07); "Violência contra o idoso" (25/07); "Violência contra a mulher - 0 que você pode fazer?" (27/07); "Violência contra a criança: Com identificar e agir" (27/07); "Principais Zoonoses urbanas: Febre amarela” (30/07); "Dia Nacional do Vira-lata" (31/07); "Principais Zoonoses urbanas: Toxoplasmose” (07/08); “Como identificar a depressão” (08/09); “Dia do Médico Veterinário” (09/09); “Divulgação da palestra Uma vida em risco! Responsabilidade pessoal?" (15/09); “Importância do autocuidado” (19/09); “Divulgação novamente da palestra Uma vida em risco! Responsabilidade pessoal?” (22/09).

Todas as publicações alcançaram juntas, até o momento, aproximadamente 12800 pessoas. 0 alcance foi observado por meios quantitativos. As "curtidas" e comentários na plataforma online foram também os parâmetros indicativos da quantidade de pessoas que reagiram às publicações realizadas. 0 principal público de alcance na plataforma foi de discentes e profissionais da área da Medicina Veterinária e também dos cursos da área da Saúde.

Foram também produzidos 9 textos por 14 anos voluntários do projeto, direcionados a profissionais de diversas áreas, como Médicos Veterinários, Assistentes Sociais, Educadores e à Prefeitura. Essa produção visa a promoção da educação sobre a relação entre a violência animal e humana, conhecida como "Teoria do Elo", e será divulgada no futuro Website "Rede Lar em Paz". Os temas abordados foram: "Maus-tratos aos animais e a violência contra a mulher"; "Maus-tratos aos animais e a violência contra crianças"; "Crianças e adolescentes cruéis com animais"; "Maustratos aos animais e crimes de outra natureza"; "A Teoria do Elo no contexto saúde única"; "A violência doméstica e a teoria do elo: identificação e atuação no contexto pedagógico"; "O assistente social e a sua importância no combate às violências presentes na teoria do elo"; "Como o médico veterinário pode agir diante da suspeita de maus-tratos aos animais"; "Para a prefeitura: o que é considerado violência doméstica e como identificar?". O site ainda está em 
desenvolvimento e em breve estará disponível, para que seja concluído o objetivo final de levar a informação da maneira mais acessível aos profissionais.

No momento, estão sendo produzidos concomitantemente dois e-books e quatro cartilhas para serem disponibilizadas futuramente na biblioteca virtual da Universidade Federal do Paraná (UFPR). Além de três textos que serão publicados no blog da Revista Clínica Veterinária. Os ebooks contam com quatro alunas voluntárias trabalhando com o tema "Violência doméstica contra o idoso, animal, criança, mulher e outras quatro participantes abordando o tema "Violência, negligência e abuso contra animais: como identificar e diferenciar cada um". Sobre as cartilhas, cada uma delas é direcionada para profissionais distintos e está sendo desenvolvida por voluntários diferentes. Elas apresentam a Identificação da Teoria do Elo para assistentes sociais, médicos veterinários, educadores e prefeituras. Os textos para Revista Clínica Veterinária visam alcançar e conscientizar os profissionais sobre "COVID - Importância da relação humano-animal em época da pandemia”, “ABANDONO - Aumento da adoção na época da pandemia” e "TEORIA DO ELO - Crianças que maltratam animais".

Foram feitos também spots de rádios, escritos por duas alunas voluntárias e encaminhados para nove estações de rádios da cidade de Curitiba e região metropolitana. Com a intenção de aumentar o alcance do projeto e atingir a comunidade externa da universidade, os spots trataram sobre a relação da COVID-19 com o aumento do abandono de animais de estimação, conscientizando o público sobre a necessidade de responsabilidade e respeito para/com todas as formas de vida. Nos próximos meses, serão desenvolvidos e divulgados mais oito spots.

\section{Cursos e palestras}

Outro produto do projeto de extensão foi o curso "Enfrentamento da violência doméstica e dos maus-tratos aos animais em tempos de COVID" que aconteceu entre agosto e outubro de 2020. 0 principal objetivo do curso foi propagar conhecimentos, através do estudo da Teoria do Elo, para possibilitar a identificação e o enfrentamento da violência por diversas categorias profissionais.

O curso foi direcionado a todos os estudantes e ministrado por profissionais das áreas de Medicina Veterinária, Psicologia, Assistência Social e Direito, teve uma carga horária efetiva de 40 horas e contou com sete módulos ofertados semanalmente, sendo eles: Módulo 1 - Ambientação e autoavaliação pré-curso; Módulo 2 - A violência na sociedade contemporânea; Módulo 3 Psicologia da violência; Módulo 4 - Aspectos legais dos maus-tratos aos animais e da violência 
doméstica; Módulo 5 - Teoria do Elo; Módulo 6 - Combate à violência doméstica e aos maus-tratos aos animais; Módulo 7 - Autoavaliação pós curso. Realizado na plataforma da UFPR Virtual.

Houve uma fase de seleção para tentar minimizar o número e evasão. Inicialmente foram recebidas 205 propostas de inscrições, então foi enviado um e-mail para todos estes com um formulário do Google que foi preenchido por 80 alunos e todos foram cadastrados na plataforma 5 dias antes do início do curso. Logo após o cadastro dos alunos.

No primeiro módulo do curso, de boas-vindas aos alunos, foi apresentada a ementa do curso, explicando como seriam liberadas as aulas semanalmente, e foram propostas atividades de apresentação, para gerar uma interação entre os mesmos. Nos demais módulos, foram ministradas aulas gravadas pelos professores que aceitaram participar do projeto de forma voluntária, e fornecidos materiais complementares às aulas para estudo. A cada semana era disponibilizada também uma atividade avaliativa com perguntas sobre a aula que foi assistida e um fórum avaliativo que objetivava a participação e interação dos alunos na resolução de casos práticos ou o compartilhamento de experiências vividas.

Ao longo do curso foram feitas análises de pré e pós exposição, análises sobre a percepção dos alunos quanto aos assuntos abordados no curso e análises sobre os motivos de evasão, para poder promover o aprimoramento do curso para futuras edições e também, promover o melhoramento de processos em cursos online e gratuitos ofertados pela plataforma UFPR Virtual.

Outra atividade realizada, foi a organização de duas palestras online, decorridas nos dias 23 e 26 de setembro, relacionadas com o tema "Setembro Amarelo", que trata sobre questões como a depressão e o suicídio. A primeira palestra ocorreu às 20 horas do dia 23 de setembro de 2020, durou quase 1 hora e 30 minutos, foi transmitida ao vivo pelo canal do YouTube da Medicina Veterinária do Coletivo e realizada através da plataforma StreamYard. 0 palestrante convidado foi o Prof. Dr. Márcio Cesar Ferraciolli, que ministrou a palestra intitulada "Uma vida em Risco! Responsabilidade pessoal?". A palestra alcançou 136 visualizações no total, sendo que atingiu um máximo de 41 visualizações síncronas, além disso, a live gravada recebeu 34 curtidas e 48 comentários A segunda palestra intitulada "Encontro virtual: a saúde mental na quarentena, uma visão Ayurvédica" foi realizada dia 26 de setembro às 10 horas e 30 minutos. Apresentada por Antonio Lacerda e Willian Bontorin, foi uma conversa interativa com o público pala plataforma GoogleMeets e contou com cerca de 30 pessoas.

Além destas atividades, está sendo organizado também um simpósio sobre Medicina Veterinária do Coletivo, programado para ocorrer em novembro deste ano, no qual serão abordados diversos temas da área, incluindo a questão atual da pandemia, e os alunos terão a 
oportunidade de desenvolverem novas habilidades a partir da participação na organização de um evento acadêmico.

\section{Considerações finais}

O projeto de extensão "Enfrentamento da Violência Doméstica e Maus-Tratos aos Animais em Tempo de COVID" está possibilitando a ampliação dos horizontes na produção e dissipação do conhecimento em relação à "Teoria do Elo" e a relação da violência interpessoal com os maustratos aos animais, para profissionais de diversas áreas e comunidade como um todo.

A partir das atividades desenvolvidas pelo projeto, foi promovida a integração de pessoas residentes nas diferentes regiões brasileiras e, consequentemente, a expansão do alcance da instituição. Mesmo sabendo que as problemáticas principal trabalhadas no projeto (a violência doméstica contra os grupos mais vulneráveis e os maus-tratos aos animais), necessitam de muitas outras ações e colaborações para serem resolvidas, é possível observar que a compreensão e conscientização da comunidade sobre o assunto é fundamental para a prevenção destas problemáticas. Assim, com base no alcance das publicações, participações no curso e nas palestras, além do feedback positivo do público sobre as ações, pode-se afirmar que, até o presente momento, os objetivos do projeto foram alcançados.

Para os alunos extensionistas participantes, os ganhos acadêmicos incluem a ampliação do conhecimento multidisciplinar, o desenvolvimento e aprimoramento de habilidades para produção de conteúdos textuais e visuais, e a oportunidade de democratizar o conhecimento acadêmico. Sendo assim, se desenvolve uma via de mão dupla, onde o estudante está encontrando a oportunidade de aplicar o conhecimento acadêmico adquirido, na forma de apoio e prestação de serviços à comunidade, ao mesmo tempo em que internaliza conhecimentos e experiências, que serão indispensáveis para seu próprio desenvolvimento acadêmico e profissional.

O objetivo das próximas etapas do projeto é expandir cada vez mais os conhecimentos para profissionais de outras áreas e para a comunidade como um todo, para que, desta forma, a conscientização possa promover uma sociedade cada vez mais apta a enfrentar as diversas formas de violência existentes.

\section{Referências}

ASCIONE, Frank R. Battered women's reports of their partners' and their children's cruelty to animals. Journal of Emotional Abuse, v. 1, n. 1, p. 119-133, 1997.

ASCIONE, Frank R.; BARNARD, S.; SELL-SMITH, J.; BROOKS, S. Animal abuse and developmental psychopathology: recent research, programmatic and therapeutic issues and challenges for the 
future. In FINE, A. H. Handbook on animal-assisted therapy: Theoretical foundations and guidelines for practice. $2^{\mathrm{a}}$ ed. Academic press, cap. 16, p. 361, 2010.

ARKOW, Phil. Human-Animal Relationships and Social Work: Opportunities Beyond the Veterinary Environment. Child and Adolescent Social Work Journal, v. 5, p. 1-16. 2020. Disponível em: <https://link.springer.com/article/10.1007/s10560-020-00697-x>. Acesso em: 24 out. 2020.

BALKIN, Diane E.; JANSSEN, Laura A.; MERCK, Melinda D. The legal system: the veterinarian's role and responsabilities. In: Veterinary Forensics: animal cruelty investigation. $2^{\underline{a}}$ ed., 2013. Disponível em:

<http://antoniogoliveira.com/site/assets/files/1260/veterinary_forensics_animal_cruelty_inves tigations-_2nd_edition.pdf $>$. Acesso em: 20 out. 2020.

BENETATO, Melanie A.; REISMAN, Robert; MCCOBB, Emily. The veterinarian's role in animal cruelty cases. Journal of the American Veterinary Medical Association, v. 238, n. 1, p. 31-34, 2011. Disponível em: <https://avmajournals.avma.org/doi/pdf/10.2460/javma.238.1.31>. Acesso em: 20 out. 2020.

BRASIL, Lei no 9.605, de 12 de fevereiro de 1998. Dispõe sobre as sanções penais e administrativas derivadas de condutas e atividades lesivas ao meio ambiente, e dá outras providências. Lei Federal, Brasília em 12 de fevereiro de 1998. Disponível em: <http://www.caurr.gov.br/wp-content/uploads/anexos/leisdecretos/lei_9605_98.pdf>. Acesso em: 22 nov. 2020.

CAMPBELL, Andrew M. An increasing risk of family violence during the Covid-19 pandemic: Strengthening community collaborations to save lives. Forensic Science International: Reports, p. 100089, 2020. Disponível em:

<https://www.sciencedirect.com/science/article/pii/S2665910720300384>. Acesso em: 22 nov. 2020.

FEDERAL, Governo. Denúncias registradas pelo Ligue 180 aumentam nos quatro primeiros meses de 2020. Ministério da Mulher, da Família e dos Direitos, 2020. Disponível em: < https://www.gov.br/mdh/pt-br/assuntos/noticias/2020-2/maio/denuncias-registradas-peloligue-180-aumentam-nos-quatro-primeiros-meses-de-2020>. Acesso em: 24 de set. 2020.

MONSALVE, Stefany; GONÇALVES, Yasmin; GARCIA, Rita. Teoria do Elo: a relação entre os maustratos aos animais e a violência interpessoal. In: GARCIA, R.C.M.; CALDERÓN, N; BRANDESPIM,

D.F. Medicina Veterinária do Coletivo: Fundamentos e Práticas. 1 ed. São Paulo: Integrativa Vet, 2019. p. 160-171.

LOCKWOOD, Randall. Counting cruelty: Challenges and opportunities in assessing animal abuse and neglect in America. The International Handbook of Animal Abuse and Cruelty: Theory, Research, and Application, p. 87-109, 2008. Disponível em: <https://www.researchgate.net/publication/301946958_Counting_Cruelty_Challenges_and_Op portunities_in_AssessingAnimal_Abuse_and_Neglect_in_America> Acesso em: 22 nov. 2020.

LOCKWOOD, Randall. Animal cruelty and human violence: the veterinarian's role in making the connection - the American experience. The Canadian veterinary jornal. v. 41, n .11, p. 876878, 2000. Disponível em: 
<https://www.ncbi.nlm.nih.gov/pmc/articles/PMC1476447/pdf/canvetj00023-0058.pdf> Acesso em: 22 nov. 2020.

MONSALVE, Stefany; FERREIRA, Fernando; GARCIA, Rita. The connection between animal abuse and interpersonal violence: a review from the veterinary perspective. Research in Veterinary Science. v. 114, p. 18-26, 2017. Disponível em:

<https://www.sciencedirect.com/science/article/pii/S0034528816305653?via\%3Dihub> Acesso em: 25 set. 2020. 
Facing domestic violence and animal abuse in times of COVID-19

\section{Abstract}

The Extension Project "Domestic Violence and Animal Abuse in COVID Time" has as objective the promotion of actions for the understanding of the Link Theory by the community, in order to create a network for the fight against domestic violence and animal abuse in the pandemic period, through the promotion of socio-educational measures through social media, websites, online courses and lectures and educational materials. All content was developed by 31 students from different areas of Brazil. From the development of the project it was remarkable the importance of disseminating such information to the community in a didactic and holistic way.
\end{abstract}

\section{Keywords}

Domestic violence; COVID-19; Link

\section{Enfrentando la violencia doméstica y el abuso animal en tiempos de COVID-19}

\section{Resumen}

El Proyecto de Extensión "Violencia Doméstica y Maltrato Animal en Tiempo de COVID", tiene como objetivo la promoción de acciones para la comprensión de la Teoría de los Vínculos por parte de la comunidad, a fin de crear una red para la lucha contra la violencia doméstica y el maltrato animal en el período pandémico, mediante la promoción de medidas socioeducativas a través de Redes Sociales, creación de sitios web, cursos y conferencias en línea y materiales educativos. Todo el contenido fue desarrollado por 31 estudiantes extensionistas de diferentes áreas del Brasil. Con el desarrollo del proyecto fue notable la importancia de difundir esa información a la comunidad de manera didáctica y holística.

\section{Palabras clave}

Violencia doméstica; COVID-19; El Link 